\section{A) Check for updates}

Cite this: Polym. Chem., 2018, 9, 490

\title{
Temperature responsive poly(phosphonate) copolymers: from single chains to macroscopic coacervates $\dagger$
}

\author{
Thomas Wolf, ${ }^{a}$ Johannes Hunold, ${ }^{\mathrm{b}}$ Johanna Simon, ${ }^{\mathrm{a}}$ Christine Rosenauer, ${ }^{\mathrm{a}}$ \\ Dariush Hinderberger (iD ${ }^{b}$ and Frederik R. Wurm (iD *a
}

\begin{abstract}
We present the first series of random poly(ethylene alkyl phosphonate) copolymers with either high solubility in water or a finely tunable hydrophilic-to-hydrophobic phase transition upon heating ("LCST"). Polymerization via the organocatalytic anionic ring-opening polymerization provided high control over molecular weight (up to $23000 \mathrm{~g} \mathrm{~mol}^{-1}$ ) and copolymer composition, and resulted in narrow molecular weight distribution $(\theta<1.3)$. Polymers of molecular weights up to $23000 \mathrm{~g} \mathrm{~mol}^{-1}$ were obtained. The phase separation temperature was precisely adjusted in a range from $55^{\circ} \mathrm{C}$ to $6{ }^{\circ} \mathrm{C}$ in water, depending on the copolymer composition. The phase transition mechanism was thoroughly investigated at different length scales via electron paramagnetic resonance spectroscopy (EPR), dynamic light scattering (DLS), UV-Vis spectroscopy and confocal laser scanning microscopy (cLSM), proving the step-wise formation of aggregates close to the cloud point temperature up to macroscopic coacervates.
\end{abstract}

Received 28th October 2017 Accepted 18th December 2017 DOI: $10.1039 / \mathrm{c} 7 \mathrm{py} 01811 \mathrm{~h}$ rsc.li/polymers solvent-polymer hydrogen bonding at elevated temperatures. ${ }^{6-8}$ As a result, the inter- and intra-chain hydrophobic-hydrophobic interactions dominate and the polymer chain collapses. This is accompanied by an increase of entropy of liberated water molecules previously bound to the polymer. ${ }^{9,10}$ In addition to the abovementioned criteria, a sharp, reversible and hysteresis-free phase transition is desirable to guarantee a fast and precise response of the material. To prevent premature reactions upon a wrong or insufficient stimulus, a precise adjustment of the phase separation temperature and detailed understanding of the underlying mechanism are essential. To this end elaborate analytical techniques (e.g. UV-Vis, EPR spectroscopy, DLS) can be used to gain a better understanding of these mechanisms on different length scales. $^{11,12}$

One class of polymers receiving increased attention as candidates in biomedical applications are poly(phosphoester)s (PPEs). ${ }^{13-17}$ The phosphoric acid ester bonds can be cleaved hydrolytically, eliminating the threat of accumulation of the polymeric material in the body. ${ }^{18-22}$ Polymers of adjustable hydrophilicity are accessible via different chemistries, and due to the pentavalent phosphorus an additional chemical functionality can be installed into every repeat unit. ${ }^{16,17,23}$ The anionic ring-opening polymerization (AROP) of cyclic phosphates gives fast access to water-soluble PPEs with high control over the molecular weight; however, transesterification reactions need to be considered..$^{24,25}$ To date, a variety of PPEs exhibiting LCST behavior have been reported. ${ }^{26-28}$ 
Recently, we developed poly(ethylene alkyl phosphonate)s (PPns) that are aliphatic poly(phosphoester)s with a phosphorus-carbon bond in the side-chain, which might be of interest for biomedical applications. These polymers are accessible via the organocatalytic ROP of 2-alkyl-2-oxo-1,3,2dioxaphospholanes providing excellent control over molecular weight and copolymer composition, and narrow molecular weight distributions. ${ }^{20,21,29}$

First studies have shown that the polymers are watersoluble, non-toxic and their properties can be finely tuned by variation of the pendant side-group..$^{20,21}$ In contrast to poly (phosphate) synthesis, transesterification side-reactions during the synthesis of poly(phosphonate)s are less pronounced, allowing living polymerization up to high conversions. ${ }^{15,20,21}$ Copolymerization of 2-alkyl-2-oxo-1,3,2-dioxaphospholanes was shown to produce random copolymers with material properties (solubility, $T_{\mathrm{g}}$ ) dependent on the copolymer composition. ${ }^{29,30}$

In this work, we study the LCST behavior of a series of novel copolymers of poly(ethylene alkyl phosphonate)s. Polymers with molecular weights between 7700 and 23000 $\mathrm{g} \mathrm{mol}^{-1}$, narrow molecular weight distributions and adjustable copolymer compositions were prepared. The phase separation temperature of the resulting copolymers was dependent on the copolymer composition. Copolymers with less than $50 \mathrm{~mol} \%$ or $30 \mathrm{~mol} \%$ of the two hydrophobic co-monomers, respectively, were water-soluble and non-thermoresponsive. Above these values phase transitions upon heating an aqueous solution was observed with adjustable macroscopic cloud points between $6{ }^{\circ} \mathrm{C}$ and $55^{\circ} \mathrm{C}$ in the current set of polymers.

To get a deeper understanding of the phase transition of these polymers, a combination of electron paramagnetic resonance spectroscopy (EPR), dynamic light scattering (DLS), UV-Vis spectroscopy, and confocal laser scanning microscopy (cLSM) was used to analyze the phase transition of these copolymers (Scheme 1). The results of this study will allow the future design of fully degradable nano- or micro-carrier devices based on PPEs.

\section{Results and discussion}

\section{Monomer synthesis}

To generate a library of temperature-responsive poly(ethylene alkyl phosphonate)s, three different five-membered cyclic phosphonates for AROP were synthesized (Scheme 2a). Monomers (1) and (2) were synthesized according to the litera-

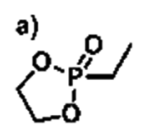

(1)

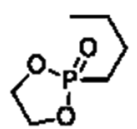

(2)

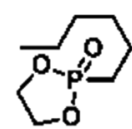

(3)

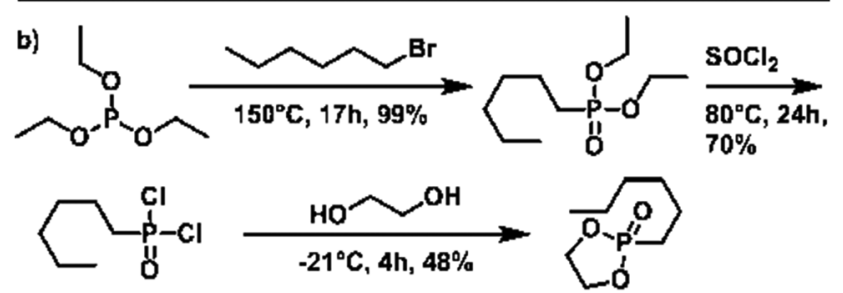

(3)

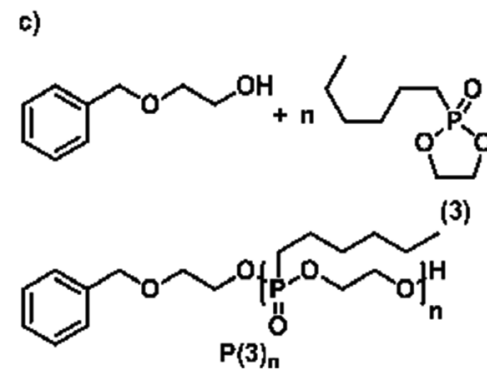

Scheme 2 (a) Structure of the 2-alkyl-2-oxo-1,3,2-dioxaphospholane monomers used in this study. (b) Synthesis scheme of 2-n-hexyl-2-oxo1,3,2-dioxaphospholane (3), starting with the Michaelis-Arbuzov reaction between triethyl phosphite and $n$-butyl bromide, followed by chlorination with thionyl chloride, and ring-closure with ethylene glycol. (c) Reaction scheme for the 2-(benzyloxy)ethanol-initiated and DBUcatalyzed AROP of (3) to produce $\mathrm{P}(3)_{n}$.

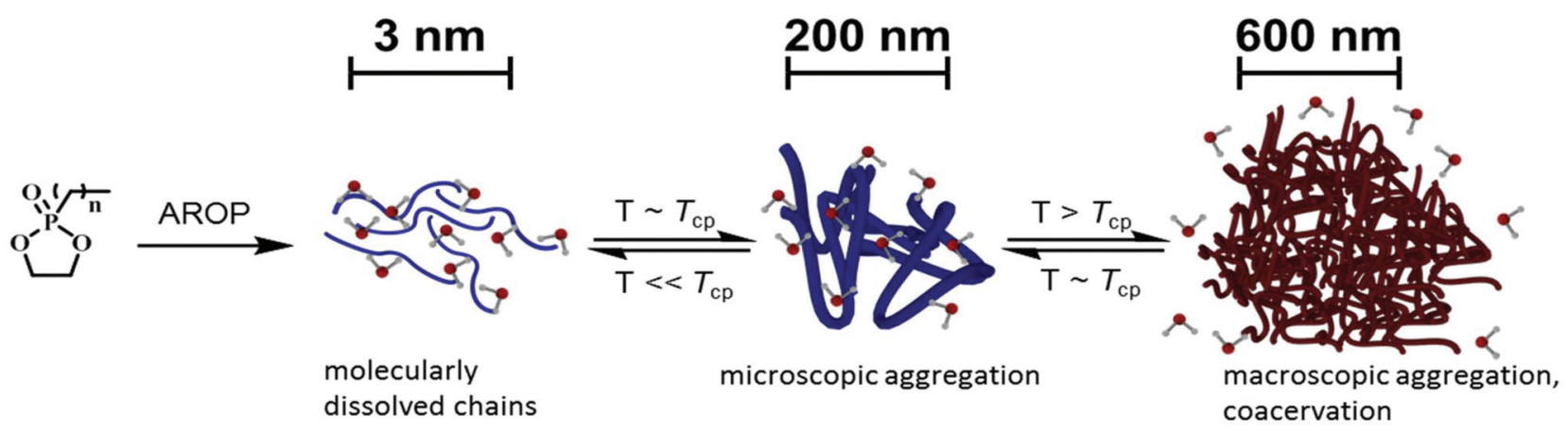

Scheme 1 Schematic presentation of this study: anionic ring-opening copolymerization produces well-defined, water-soluble random copolymers. Phase separation behavior of the copolymers in the aqueous medium is investigated through various methods. Formation of nano-inhomogeneities and microscopic aggregates close to the cloud point temperature $\left(T_{\mathrm{cp}}\right)$ observed via CW EPR and DLS measurements, respectively. Macroscopic aggregation above the $T_{\mathrm{cp}}$ and the formation of coacervates visualized via turbidity measurements and confocal laser scanning microscopy. 
ture. ${ }^{20}$ Monomer (3) with a hydrophobic $n$-hexyl side-chain was newly developed for the current study (Scheme 2b): the Michaelis-Arbuzov reaction of $n$-hexyl bromide and triethyl phosphite yielded $O, O$-diethyl $n$-hexyl phosphonic acid diethyl ester. Conversion to the respective phosphonic acid dichloride was achieved by chlorination with thionyl chloride. Finally, condensation with ethylene glycol provided the cyclic monomer (3). Detailed experimental description, as well as analytical data, can be found in the ESI (Fig. S1-S6†).

\section{Homopolymerization}

First, the polymerization behavior of 2- $n$-hexyl-2-oxo-1,3,2-dioxaphospholane (3) as well as the respective properties of $\mathbf{P}(3)_{n}$ were investigated. Following previously reported results regarding the AROP of dioxaphospholanes, ${ }^{n}$ HexPPn was polymerized in dichloromethane (DCM) with 1,8-diazabicyclo-[5.4.0]undec7-ene (DBU) as a catalyst at $30{ }^{\circ} \mathrm{C}$ with 2 -(benzyloxy)ethanol as an initiator, which allowed end-group analysis via ${ }^{1} \mathrm{H}$ NMR spectroscopy (Scheme 2c). ${ }^{20}$

Successful polymerization was indicated from the ${ }^{1} \mathrm{H}$ NMR spectra by the broad resonance of the backbone protons ranging from 4.22 to $4.01 \mathrm{ppm}$ after $17 \mathrm{~h}$ of polymerization time (Fig. 1a, signal c). $M_{\mathrm{n}}$ was determined via end-group analysis, by a comparison of the initiator signals at $7.26 \mathrm{ppm}$ with the backbone resonances. The accuracy of this method is limited for high molecular weight polymers, but has been proven for PPns with $P_{\mathrm{n}}$ up to $200 .{ }^{21}$ The ${ }^{31} \mathrm{P}\{\mathrm{H}\}$ spectrum (Fig. 1a, inset) reveals a distinct shift from the monomer (51.4 ppm) to a polymer with two phosphorus signals originating from the backbone (Fig. 1, signal 1, $33.5 \mathrm{ppm}$ ) and the terminal phosphorus (Fig. 1, signal 1', 32.9 ppm).

SEC analysis (Fig. 1b) shows symmetrical monomodal molecular weight distributions ( $D \sim 1.20$; vs. PEG standards). The molecular weight of the polymer was determined from NMR spectra, but not via SEC, as the solvation behavior and dynamic radii between the PEG standard and PPns are very
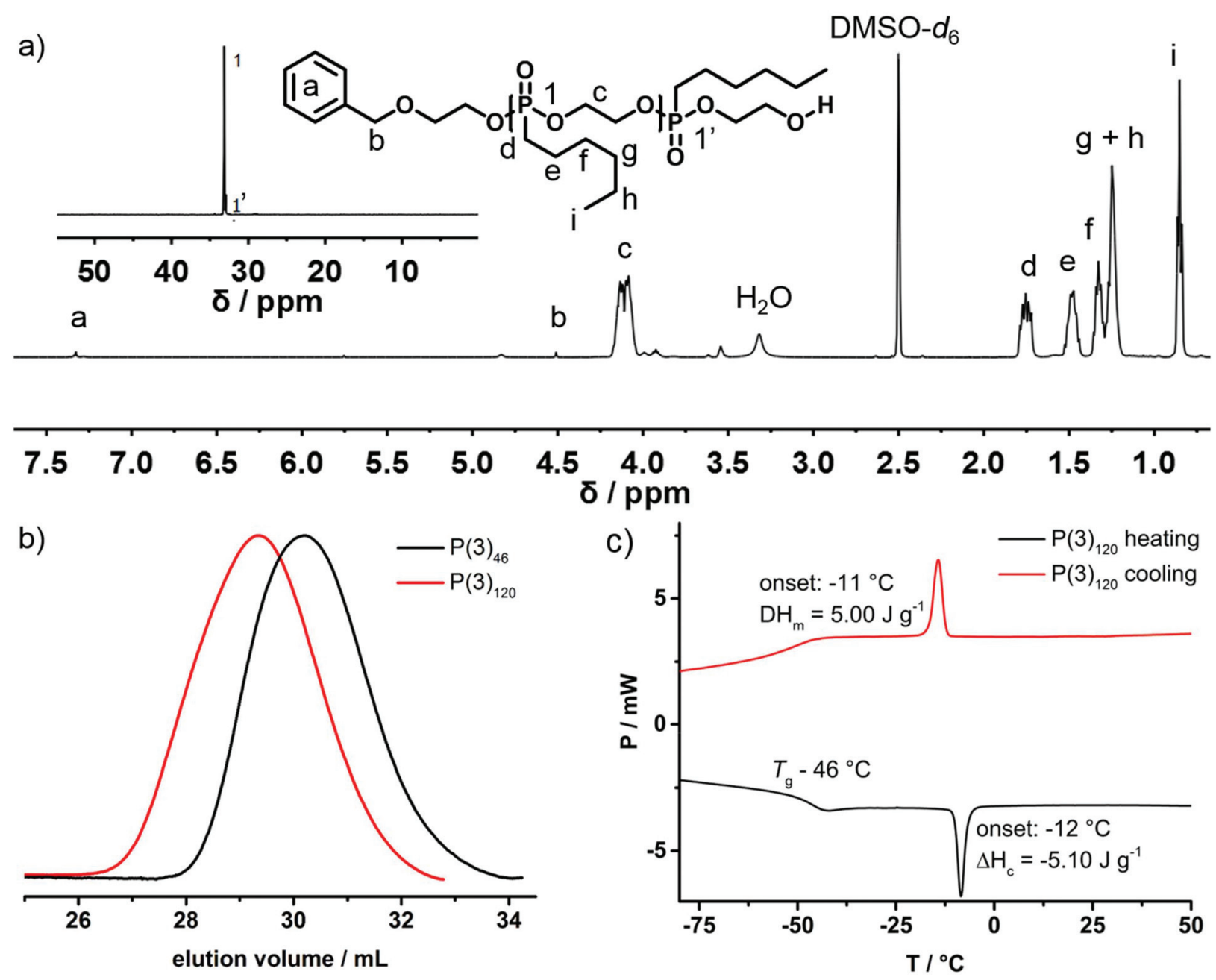

Fig. 1 (a) ${ }^{1} \mathrm{H} N M R(500 \mathrm{MHz})$ and ${ }^{31} \mathrm{P}\{\mathrm{H}\}$ NMR $(201 \mathrm{MHz})$ (inset) spectra of $\mathrm{P}(3)_{120}$ in DMSO- $d_{6}$ at $298 \mathrm{~K}$. (b) SEC elugrams (RI detection) of $\mathrm{P}(3)_{120}$ and $\mathrm{P}(3)_{46}$ in DMF at $333 \mathrm{~K}$. (c) DSC thermogram of $\mathrm{P}(3)_{120}$ at a heating rate of $10^{\circ} \mathrm{C} \mathrm{min}^{-1}$ in the temperature range from $-80{ }^{\circ} \mathrm{C}$ to $50{ }^{\circ} \mathrm{C}$ (second heating and cooling curves shown). 
different. The polymer is insoluble in water but can be readily dissolved in organic solvents such as diethyl ether, dichloromethane, chloroform, dimethyl sulfoxide, or dimethylformamide. Thermal analysis via DSC (Fig. 1c) reveals a low glass transition temperature $\left(T_{\mathrm{g}}=-46^{\circ} \mathrm{C}\right)$ similar to those of previously reported poly(alkylene alkyl phosphonate)s. Additionally, a melting signal with the onset at $-10^{\circ} \mathrm{C}$ and an enthalpy of fusion of $5 \mathrm{~J} \mathrm{~g}^{-1}$ is observed, indicating the first side-chain crystallization observed for poly(ethylene alkyl phosphonates)s from AROP. ${ }^{20,21}$ The polymerizability of monomer (3) ensured that we proceeded with the synthesis of the thermoresponsive poly(ethylene alkyl phosphonate) copolymers $\mathbf{P}\left(\mathbf{1}_{\boldsymbol{x}}-\boldsymbol{c o}-\mathbf{2}_{\boldsymbol{y}}\right)$ and $\mathbf{P}\left(\mathbf{1}_{\boldsymbol{x}}-\boldsymbol{c o}-\mathbf{3}_{\boldsymbol{y}}\right)$.

\section{Copolymerization}

The organocatalytic anionic ring-opening copolymerization of monomer (1), resolution in hydrophilic repeat units, with either monomer (2) or (3) (each producing hydrophobic units) was performed at $30^{\circ} \mathrm{C}$ with DBU as the respective catalyst in a one-pot reaction for $17 \mathrm{~h}$ (Scheme 3 ).

The representative ${ }^{1} \mathrm{H}$ and ${ }^{31} \mathrm{P}\{\mathrm{H}\}$ NMR spectra of $\mathbf{P}\left(\mathbf{1}_{\boldsymbol{x}}\right.$-co$\mathbf{2}_{\boldsymbol{y}}$ ) and $\mathbf{P}\left(\mathbf{1}_{\boldsymbol{x}}-\boldsymbol{c o}-\mathbf{3}_{\boldsymbol{y}}\right)$ are shown in Fig. 2. Molecular weights can be determined by the end-group analysis of the ${ }^{1} \mathrm{H}$ NMR spectra (vide supra). Polymers with molecular weights ranging from 7700 to $23000 \mathrm{~g} \mathrm{~mol}^{-1}$ were obtained. ${ }^{1} \mathrm{H}$ NMR spectroscopy further provided information on the copolymer composition in the final material.

a)

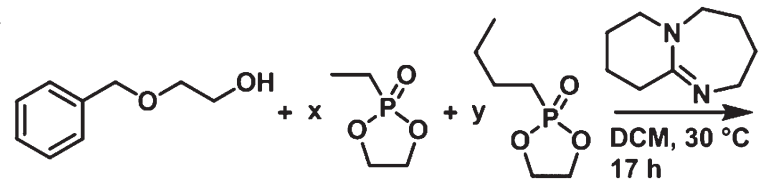

(1)

(2)

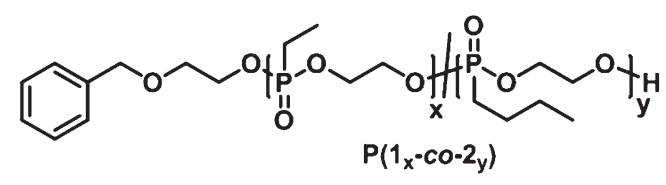

b)

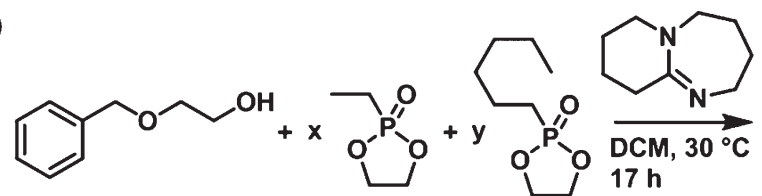

(1)

(3)

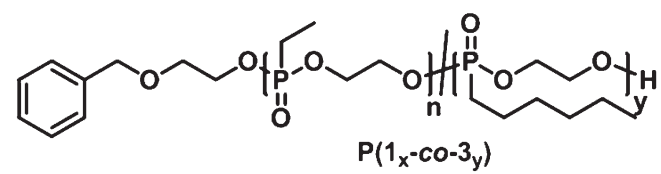

Scheme 3 Reaction scheme for the 2-(benzyloxy)ethanol-initiated and DBU catalyzed anionic ring-opening copolymerization of (1) with (a) (2) to form $\mathrm{P}\left(1_{x}-\mathrm{co}-2_{y}\right)$ and with (b) (3) to form $\mathrm{P}\left(1_{x}-\mathrm{co}-3_{y}\right)$.
The resonance of the terminal $-\mathrm{CH}_{3}$ side-chains of (1) at $1.06 \mathrm{ppm}$ is well separated from the respective terminal $-\mathrm{CH}_{3}$ group resonances of (2) and (3) at $0.86 \mathrm{ppm}$, allowing the calculation of the copolymer composition. In addition to ${ }^{1} \mathrm{H}$ NMR spectroscopy, the comparison of the relative intensities of the backbone resonances in the ${ }^{31} \mathrm{P}\{\mathrm{H}\} \mathrm{NMR}$ spectra at $34.4 \mathrm{ppm}$ for (1) and $33.2 \mathrm{ppm}$ for (2) and (3), respectively, also reveals the copolymer composition (Fig. 2, insets). The experimentally found polymer composition matched the monomer feed ratios in all cases, both from ${ }^{1} \mathrm{H}$ or ${ }^{31} \mathrm{P}$ NMR analysis. ${ }^{1} \mathrm{H}$ DOSY NMR spectra (Fig. S10†) further prove successful copolymerization, as all relevant resonances, i.e. -backbone and respective sidechains, have the same diffusion coefficient and are part of the same molecule. All polymers regardless of molecular weight or copolymer composition showed symmetrical monomodal SEC traces with rather narrow molecular weight distribution $(1.18<$ $Ð<1.30$; vs. PEG standard) (Fig. S12 and S14 $\dagger$ ).

The microstructure of the copolymers is expected to have a significant influence on the solubility and phase separation behavior. Therefore, the copolymerization kinetics of (1) with (2) and (3), respectively, were analyzed via ${ }^{31} \mathrm{P}\{\mathrm{H}\}{ }^{\mathrm{NMR}}$ spectroscopy.

Fig. S15† shows the molar fraction of (1) integrated into the respective copolymer at different times during the copolymerization. Starting from the initial 1:1 ratio of the monomer feed $([\mathrm{A}] /([\mathrm{A}]+[\mathrm{B}])=0.5)$, the copolymer composition remains constant for the whole duration of both copolymerizations. This indicates that all co-monomers are incorporated in the copolymer at the same rate and have the same reactivity, thus resulting in the formation of a random copolymer without any detectable gradients.

DSC analysis shows that the side-chain crystallinity observed for $\mathbf{P}(\mathbf{3})_{\mathbf{1 2 0}}$ vanishes for the copolymers resulting in completely amorphous materials (Fig. S16 and S17†). Cell-toxicity measurements against the macrophage cell-line RAW 264.7 showed a concentration-dependent cell-toxicity for $\mathbf{P}\left(\mathbf{1}_{\mathbf{5 1}}\right.$ co-2 $\left.{ }_{51}\right)$ and $\mathbf{P}\left(\mathbf{1}_{41}-\boldsymbol{c o}-\mathbf{3}_{16}\right)$. The toxicity was negligible for pharmaceutically suitable concentrations $\left(<100 \mu \mathrm{g} \mathrm{mL}^{-1}\right)$. Above $250 \mu \mathrm{g} \mathrm{mL} \mathrm{m}^{-1}$, a reduction in the viability is observed (Fig. S18†). The effect was more pronounced for the more hydrophobic copolymers $\mathbf{P}\left(\mathbf{1}_{\mathbf{4 1}}-\boldsymbol{c o}-\mathbf{3}_{\mathbf{1 6}}\right)$. A similar trend was previously reported for other poly(ethylene alkyl phosphonate)s with hydrophobic side-chains. ${ }^{20}$ The analytical data of all copolymers are summarized in Tables S1 and S2. $\dagger$

\section{Thermal behavior in solution}

The solubility and thermal behavior of the copolymers in water were thoroughly investigated. First, turbidity measurements were performed to investigate the macroscopic solution behavior. Copolymers with low amounts of the hydrophobic comonomers exhibited high solubility in water $\left(>10 \mathrm{~g} \mathrm{~L}^{-1}\right)$; however, polymers containing more than $50 \mathrm{~mol} \%$ of (2) or $30 \mathrm{~mol} \%$ of (3), respectively undergo a phase separation upon heating. The cloud point temperatures $\left(T_{\mathrm{cp}}\right)$ were tailored over a broad temperature range from $6{ }^{\circ} \mathrm{C}$ to $55{ }^{\circ} \mathrm{C}$ in the current copolymer set (Fig. 3a and b). A linear dependency between 

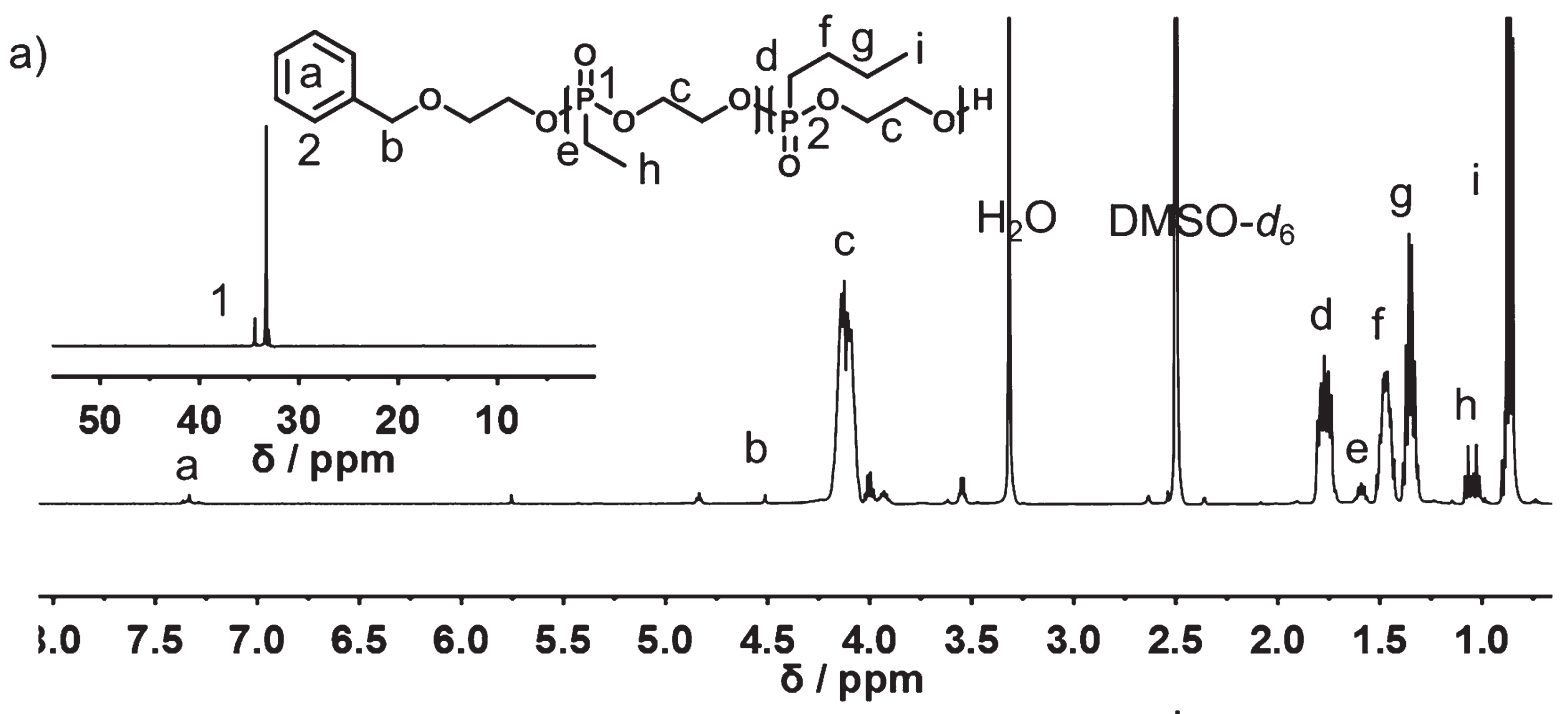

b)
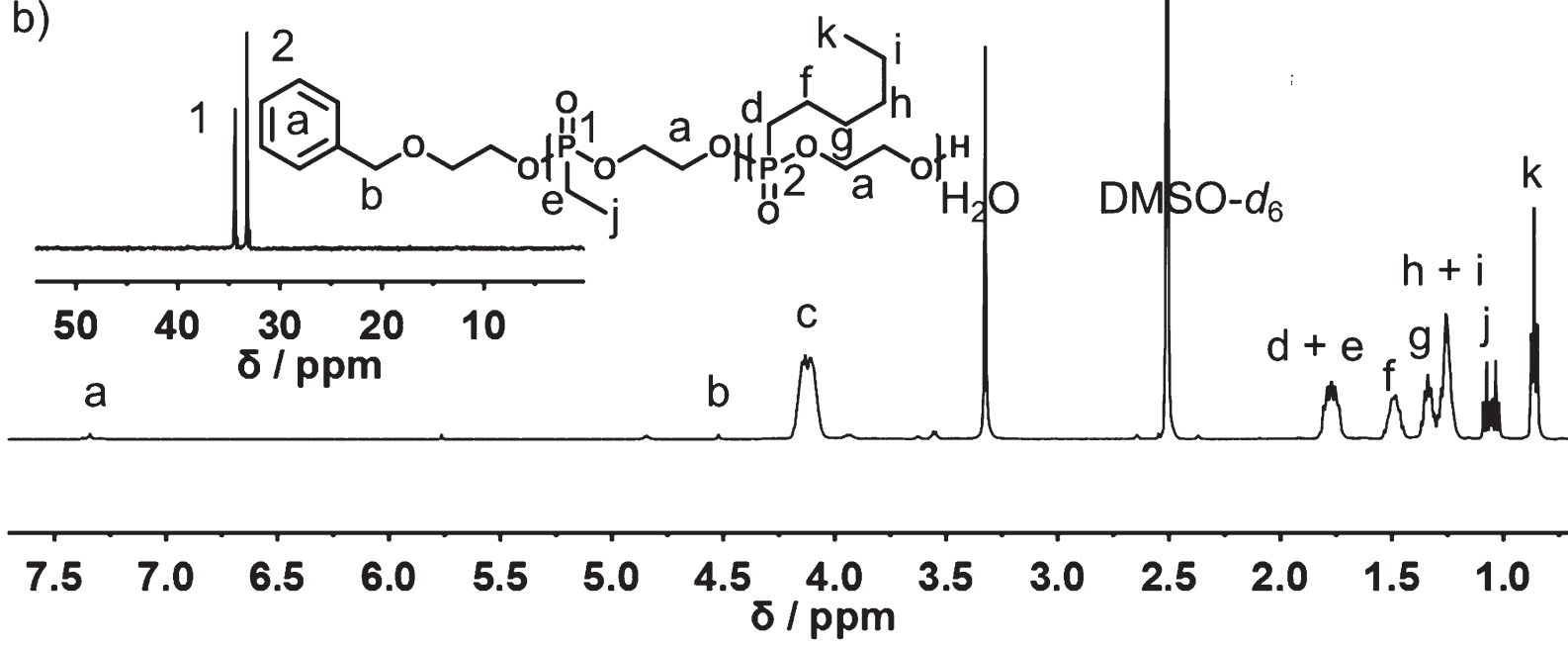

Fig. $2{ }^{1} \mathrm{H}$ NMR $(500 \mathrm{MHz})$ and ${ }^{31} \mathrm{P}\{\mathrm{H}\} \mathrm{NMR}\left(201 \mathrm{MHz}\right.$, inset) spectra of (a) $\mathrm{P}\left(1_{15}-c 0-2_{118}\right)$ and (b) $\mathrm{P}\left(1_{62}-c 0-3_{56}\right)$ in DMSO-d at $298 \mathrm{~K}$.

the $T_{\mathrm{cp}}$ and a number of hydrophobic side-chains incorporated in the copolymer was found (Fig. 3c). In all cases, little to no hysteresis occurred during the cooling of the samples, indicating a well-reversible phase transition.

In accordance with the literature, the LCST cloud point temperatures were found to be dependent on the ionic strength of the solution. ${ }^{31}$ The addition of sodium phosphate (0.1 M PBS, pH 7.4) shifted the phase transition by $6^{\circ} \mathrm{C}$ to lower temperatures (Fig. S19†). This is in good accordance with the theory of Hofmeister et al. with regard to the antichaotropic effect of sodium ions. ${ }^{31}$

To gain a more detailed understanding of the phase separation behavior of poly(ethylene alkyl phosphonate)s, three additional methods were used, each using different length scales during the phase separation process: EPR spectroscopy was employed to detect the formation of nanodimensional structural inhomogeneities. Then, dynamic light scattering was employed to analyze the formation of larger aggregates prior to the macroscopic precipitation of the solution visible by UV-Vis turbidity measurements. Finally, confocal laser scanning microscopy visualized the macroscopic aggregates and analyzed their structures.

The CW EPR spectra recorded during the heating of an aqueous solution of $15 \mathrm{mg} \mathrm{mL} \mathrm{m}^{-1}$ of $\mathbf{P}\left(\mathbf{1}_{33}-\boldsymbol{c o}-\mathbf{2}_{76}\right)$ with $0.2 \mathrm{mM}$ 2,2,6,6,-tetramethylpiperidin-1-oxyl (TEMPO) in DPBS buffer are shown in Fig. 3d and e. Under these conditions, which were optimized for the CW EPR measurements, macroscopic phase separation occurred at $25{ }^{\circ} \mathrm{C}$. EPR spectroscopy is a method for studying materials with an unpaired electron, like the spin probe TEMPO. The method is sensitive towards the chemical environment of the probe and minuscule changes in, e.g., hydrophilicity of the surrounding medium can be detected and characterized. ${ }^{32-34}$ It can be seen in Fig. 3 that the intensity and the line shape of the high-field peak change with increasing temperature. In the range between 10 and $16^{\circ} \mathrm{C}$, the intensity of the high-field line first decreases slightly when the temperature increases, which could e.g. indicate a slight rise of the nanoviscosity around the spin probe or a 
a)

b)
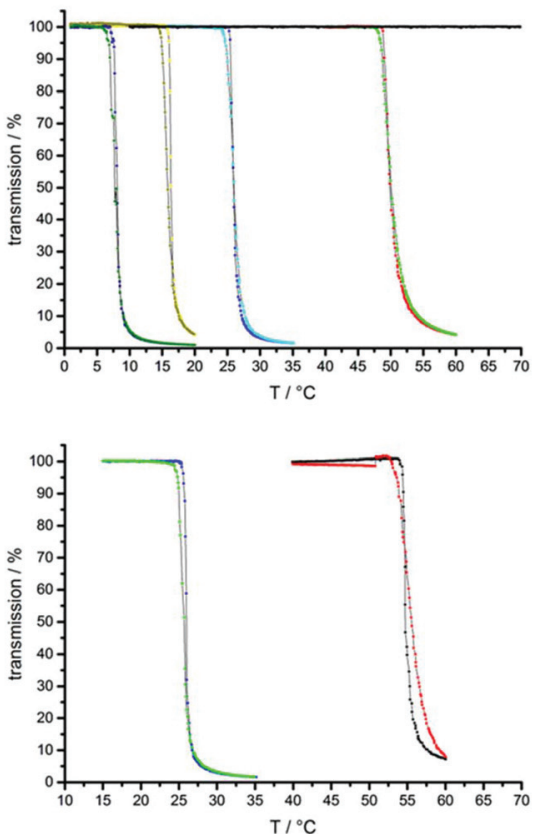

c)

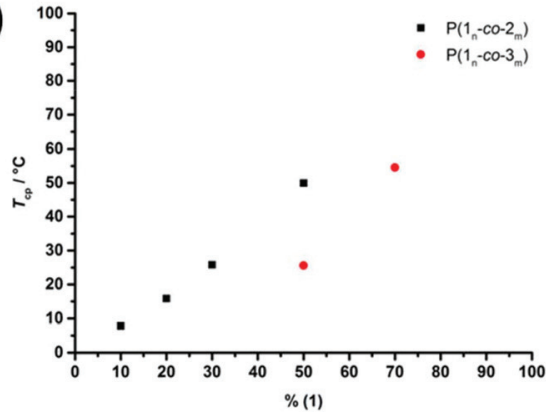

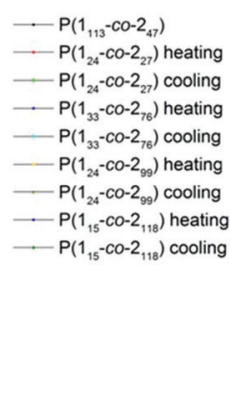

- $\mathrm{P}\left(1_{4},-c 0-3_{16}\right)$ heating - $\mathrm{P}\left(1_{41},-\mathrm{co}-3_{16}\right)$ cooling - $\mathrm{P}\left(1_{32}-\mathrm{co}-3_{30}\right)$ cooling - $\mathrm{P}\left(1_{32}{ }^{-} \mathrm{CO}-3_{30}\right)$ heating (a)
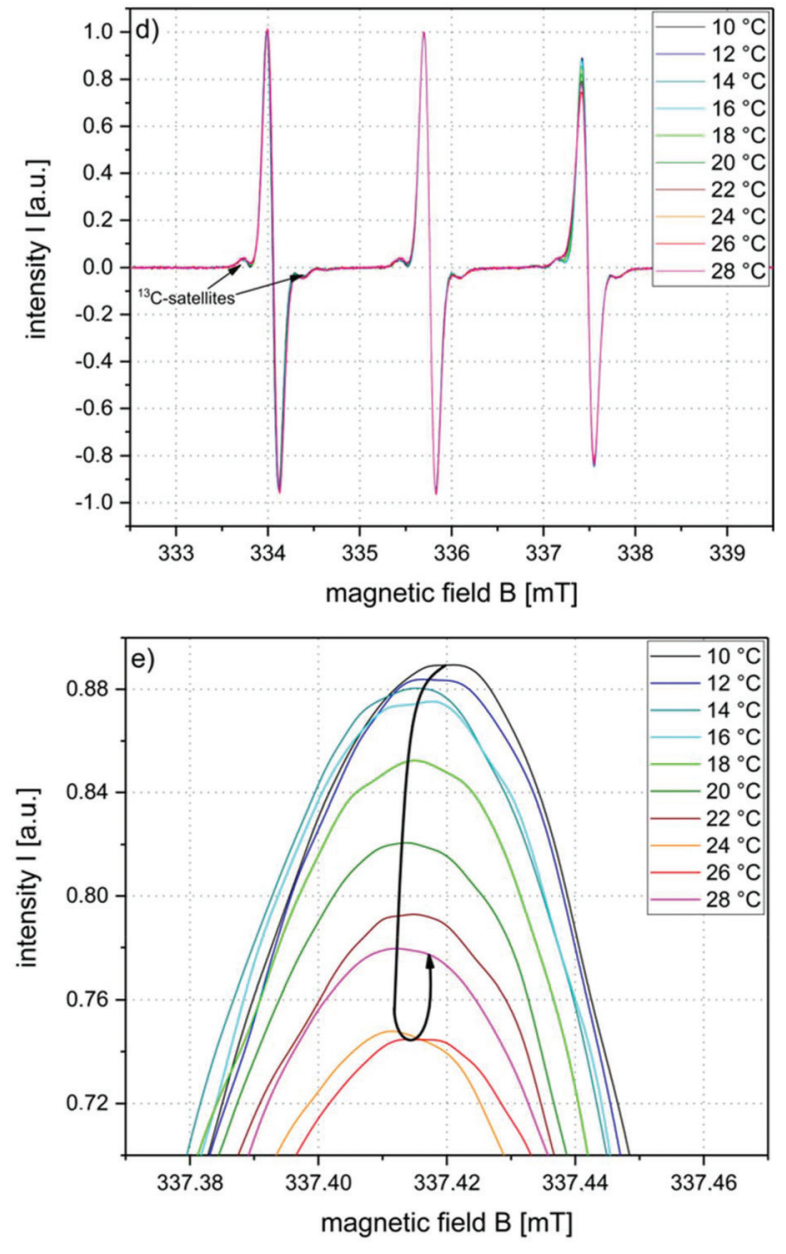

Fig. 3 Thermal response of poly(ethylene alkyl phosphonate) copolymers: turbidity measurements (heating and cooling curves) of (a) $\mathrm{P}\left(\mathbf{1}_{\mathrm{x}}-\mathrm{co}-\mathbf{2}_{y}\right)$ and (b) $\mathrm{P}\left(1_{x}-\mathrm{co}-3_{y}\right)$ in ultrapure water at a concentration of $10 \mathrm{~g} \mathrm{~L}^{-1}$ and a heating/cooling rate of $1{ }^{\circ} \mathrm{C} \mathrm{min}{ }^{-1}$. The transmission was measured at $500 \mathrm{~nm}$. (c) Correlation between comonomer ratio and $T_{\mathrm{cp}}$. CW EPR spectra of an aqueous solution of $15 \mathrm{mg} \mathrm{mL}^{-1} \mathrm{P}\left(1_{33}-\mathrm{co}-2_{76}\right)$ with $0.2 \mathrm{mM}$ TEMPO in DPBS buffer. (d) Full spectra and (e) enlarged high-field region (full high-field peak see Fig. S21†).

slight decrease in polarity in the spin probe environment experienced by some of the spin probes.

If, however, the temperature is only increased further by just $2{ }^{\circ} \mathrm{C}$ (from 16 to $18{ }^{\circ} \mathrm{C}$ ), the signal intensity maximum decreases significantly. The reason for the observed intensity decrease of the high-field line is the simultaneous appearance of a second, slightly less polar solvated, probe species at $18{ }^{\circ} \mathrm{C}$. This spin probe species shows a slightly smaller hyperfine splitting constant A (i.e. its high-field line appears at slightly lower magnetic field values) indicating a lower spin probe environmental polarity. Since the hyperfine coupling values of this second, "hydrophobic", species ( $\sim 7 \mathrm{MHz})$ are still very high compared to those of these species in other, more conventional LCST type polymers $(\sim 44-45 \mathrm{MHz})$, we can conclude that the less polar polymer-rich regions, probed by the appearance of the second spin probe species, are still water swollen (A in bulk water: $\sim 48 \mathrm{MHz}$ ). ${ }^{11,32-34}$ As seen in these other LCST-type polymers, CW EPR spectroscopy on TEMPO can detect small, nanoscopically dehydrated regions at tempera- tures below the cloud point. ${ }^{11}$ Under the same conditions, no macroscopic aggregation was observed in dynamic light scattering measurements, even at $20{ }^{\circ} \mathrm{C}$ (Fig. S20†). This shows that our EPR spectroscopic scheme adds molecular insights that DLS cannot provide for the investigation of the phase separation close to the LCST.

In these LCST polymers, we can distinguish two types of water: one is bulk water with the relatively freely tumbling hydrophilic probe species with comparatively high environmental polarity. The other one is polymer-associated water that forms the hydration shell around the polymer chains where the more hydrophobic probe species with a reduced environmental polarity is located. Thus, at $6-7{ }^{\circ} \mathrm{C}$ below the macroscopic cloud point temperature $T_{\mathrm{cp}}$ of about $25{ }^{\circ} \mathrm{C}$ a phase separation between still fully solvated and less solvated yet still water-swollen polymer regions on the nanoscale could be observed by EPR spectroscopy. These nanoscopic inhomogeneities continue to grow at a further temperature increase so that the polymer chains are more and more dehydrated (and 
yet still water swollen in comparison with conventional LCST polymers). As an additional result of this dehydration process, the effective nanoviscosity of the spin probe (i.e. the viscosity, which the probe senses in a radius of about $2-3 \mathrm{~nm}$ around it) increases, and also its mobility is reduced. Both effects together lead to a steady signal intensity decrease of the highfield peak, until it reaches its minimum at $24-26^{\circ} \mathrm{C}$, which corresponds quite well to the $T_{\mathrm{cp}}$. Considering that the TEMPO probes have a van der Waals-radius of about 0.31-0.35 nm (ref. 35) the EPR spectroscopically detected nanoscopic inhomogeneities should not be much larger in diameter than about 5-6 nm. Otherwise, these inhomogeneities would no longer be perceived as such by the relatively small TEMPO probes. ${ }^{11,32}$

A temperature increase beyond $26{ }^{\circ} \mathrm{C}$ leads to a renewed increase in the signal intensity of the high-field peak. This indicates that the polymer chains are further dehydrated (as the phase separation process progresses with the temperature increase), but now some of the hydrophobic probe species are expelled from the inhomogeneities along with polymer-associated water. Consequently, the (spectral) proportion of relatively freely tumbling hydrophilic spin probe species and also the signal intensity of the high field peak increase again.

To further investigate the aggregation during the phase separation process, temperature-dependent DLS measurements were performed. $\mathbf{P}\left(\mathbf{1}_{\mathbf{2 4}}-\boldsymbol{c o}-\mathbf{2}_{27}\right)$ with the $T_{\mathrm{cp}}$ at $50{ }^{\circ} \mathrm{C}\left(10 \mathrm{~g} \mathrm{~L}^{-1}\right.$, $\mathrm{H}_{2} \mathrm{O}$ ) was chosen for these experiments, as precise heating of the DLS cell was only viable in a temperature range from 30 to $60{ }^{\circ} \mathrm{C}$ in our setup. Fig. 4a shows the hydrodynamic radii measured in the light scattering experiment (left, black) and a)
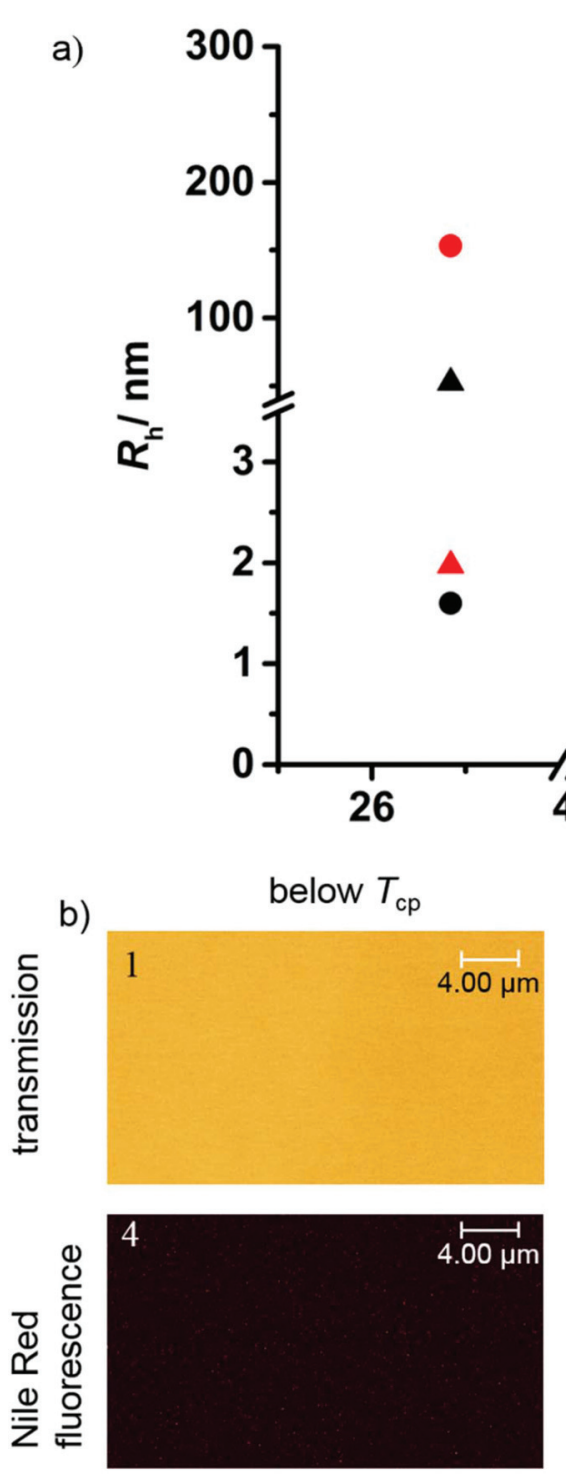

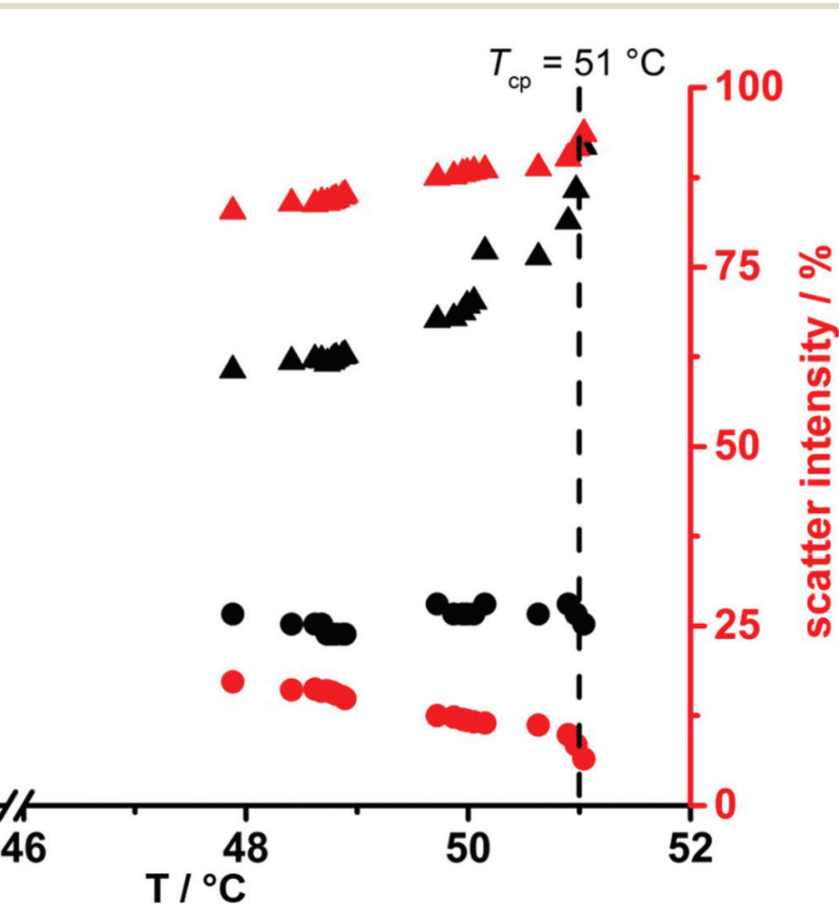

$\mathbf{T} /{ }^{\circ} \mathbf{C}$

above $T_{\text {cp }}$ for $10 \mathrm{~s}$
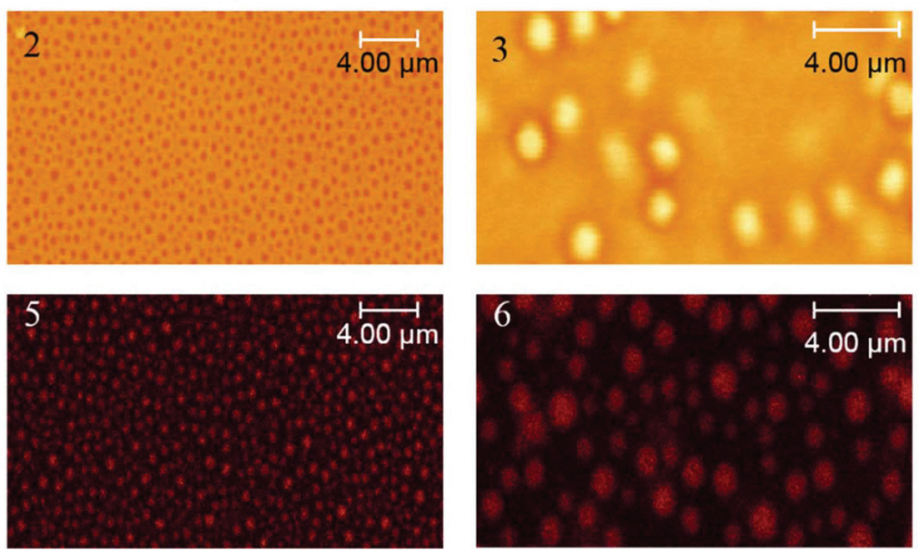

Fig. 4 (a) Temperature-dependent DLS measurements of $\mathrm{P}\left(1_{24}-c 0-2_{27}\right)$. (b) Confocal laser scanning microscopy pictures of $\mathrm{P}\left(\mathbf{1}_{15}-\mathrm{co}-\mathbf{3}_{118}\right)\left(T_{\mathrm{cp}}=\right.$ $8{ }^{\circ} \mathrm{C}$ ): top row: transmission channel below the $T_{\mathrm{cp}}$ (1), after surpassing the $T_{\mathrm{cp}}$ (2) and above the $T_{\mathrm{cp}}$ (3); bottom row: respective Nile Red fluorescence channel below (4), after surpassing (5) and above the $T_{c p}(6)$. 
the relative intensities (right, red) of the observed processes as a function of temperature. At $27^{\circ} \mathrm{C}$, below the $T_{\mathrm{cp}}$, the majority of the polymers are molecularly dissolved with a small fraction of aggregates only. This can be concluded as there are only two processes observed: one process with a hydrodynamic radius of $1.5 \mathrm{~nm}$ corresponding to $70 \%$ of the total scattering intensity (molecularly dissolved polymer, circles) and the other process with a hydrodynamic radius of $50 \mathrm{~nm}$ corresponding to $30 \%$ of the scattering intensity (aggregates, triangles). Due to the strong size dependence of scattering intensity $(I \propto r 3)$ the latter process scatters nearly 40000 times stronger than the $1.5 \mathrm{~nm}$ process. Therefore, below $T_{\mathrm{cp}}$, a negligible fraction of the polymer is already present in an aggregated state while the majority is molecularly dissolved. Upon heating, the relative scattering intensities of these processes change when approaching the phase separation temperature: while keeping a constant radius, the scattering intensity of the $1.5 \mathrm{~nm}$ process drops to $12 \%$ at $50{ }^{\circ} \mathrm{C}$. The radius and scattering intensity of the second process, however, gradually increase to $120 \mathrm{~nm}$ and $88 \%$, respectively at $50{ }^{\circ} \mathrm{C}$. Upon surpassing the $T_{\text {cp }}$ a sharp and fast increase of the radius is observed up to large aggregates of $R_{\mathrm{h}}>250 \mathrm{~nm}$.

Such large structures are easily observed by confocal laser scanning microscopy (cLSM). The respective cLSM pictures of $\mathbf{P}\left(\mathbf{1}_{15}-\boldsymbol{c o}-\mathbf{3}_{118}\right)$ at different temperatures are shown in Fig. $4 \mathrm{~b}$. The investigated polymer was switched to a more hydrophobic polymer with a $T_{\mathrm{cp}}$ of $8{ }^{\circ} \mathrm{C}$ as the microscope could not be heated and needed to be cooled down during the measurement. In addition to conventional transmission spectroscopy to observe the structures of the aggregates the fluorescence of Nile Red, a solvatochromic dye with weak fluorescence in hydrophilic medium and strong fluorescence in a more hydrophobic environment, was measured. For this purpose, Nile Red was co-dissolved in the aqueous polymer solution below $T_{\mathrm{cp}}$ and heated during the measurement. The concentration of the dye was kept as low as possible $\left(1 \mu \mathrm{g} \mathrm{mL} \mathrm{m}^{-1}\right)$ to prevent interactions between the responsive polymer and the dye. However, even at considerably higher concentrations, no change in the solubility behavior of the polymer was observed. Below the $T_{\mathrm{cp}}$ of the copolymer, a clear solution with little fluorescence was observed in the transmission and Nile Red channel, indicating a homogeneous hydrophilic environment under this resolution. This indicates that the subtleties of pre-collapsed dehydration as seen in CW EPR are not detectable with the much larger and more hydrophobic probes. Yet, to characterize the large aggregates formed, this method complements the few nm scale characterization by EPR spectroscopy. If the temperature is raised above the $T_{\mathrm{cp}}$ of the polymer well-defined spherical structures with diameters of $c a$. $600 \mathrm{~nm}$ abruptly become visible. These structures show strong Nile Red fluorescence indicating a less hydrophilic environment compared to the surrounding water phase. This is in good accordance with the observation of $R_{\mathrm{h}}=250 \mathrm{~nm}$ aggregates observed from DLS measurements. These aggregates grew in size over time due to coalescence and eventually formed perfectly spherical coacervate droplets with a diameter of $c a .2000 \mathrm{~nm}$, as already observed for a related system. ${ }^{30}$

With these results, we can propose a mechanism for the LCST phase separation of these poly(ethylene alkyl phosphonate) copolymers.

At low temperatures, the major fraction of the copolymer is molecularly dissolved in water as unimers. Upon increasing the temperature, the steadily decreasing polymer-water hydrogen bond interaction results in the formation of nanoscopic polymer inhomogeneities, as visualized by CW EPR spectroscopy. A further increase in temperature leads to complete dehydration of the polymer and the formation of aggregates $\left(R_{\mathrm{h}}<100 \mathrm{~nm}\right)$ is detectable by DLS. These slowly increase in size till a threshold temperature is reached upon which a rapid phase separation occurs into a polymer free water-phase and large polymer aggregates $\left(R_{\mathrm{h}} \sim 250 \mathrm{~nm}\right)$. Macroscopically, this results in the clouding of the solution and the detection of $T_{\mathrm{cp}}$ by transmission spectroscopy. Over time, coalescence of the aggregates results in the formation of large macroscopic droplets and complete phase separation.

\section{Conclusions}

In summary, we studied the hydrophilic-to-hydrophobic phase transition of thermo-responsive aliphatic poly(phosphonate)s on different length scales: the nanometer-sized regime was studied by CW EPR spectroscopy, revealing two types of water: first, bulk water with the freely tumbling hydrophilic probe and second, polymer-associated water that forms the hydration shell around the polymer chains where the more hydrophobic probe species with a reduced environmental polarity is located. Upon reaching/exceeding a critical temperature, fast aggregate growth and the formation of macroscopic droplets were observed (by dynamic light scattering and confocal microscopy) resulting in the clouding of the solution (quantified by turbidity measurements). The LCST behavior was studied based on a series of poly(ethylene alkyl phosphonate) copolymers, which were prepared by the ring-opening polymerization of 2-alkyl-2-oxo-1,3,2-dioxaphospholanes with ethyl, butyl, and hexyl side chains. Random copolymers with molecular weights between 7700 and $23000 \mathrm{~g} \mathrm{~mol}^{-1}$ and molecular weight distributions between 1.18 and 1.30 were prepared, which exhibited a thermo-responsive behavior over a broad temperature range depending on the copolymer composition. The results of this study give a deeper insight into the LCST phase transition in PPE-copolymers and will allow the future design of fully degradable thermo-responsive materials based on PPEs.

\section{Conflicts of interest}

There are no conflicts to declare. 


\section{Acknowledgements}

The authors thank Angelika Manhart (MPIP) for synthetic assistance, Manfred Wagner (MPIP) for NMR measurements, Anke Kaltbeitzel (MPIP) for assistance with the cLSM measurements, and Franziska Zeuner and Heike Schimm (both MLU) for technical support. The authors acknowledge the support from the "Deutsche Forschungsgemeinschaft" (DFG WU 750/61) and the Fonds der Chemischen Industrie (FCI, JH and DH). Open Access funding provided by the Max Planck Society.

\section{References}

1 B. D. Ratner and S. J. Bryant, Annu. Rev. Biomed. Eng., 2004, 6, 41-75.

2 S. R. Meyers and M. W. Grinstaff, Chem. Rev., 2012, 112, 1615-1632.

3 S. Anastase-Ravion, Z. Ding, A. Pelle, A. S. Hoffman and D. Letourneur, J. Chromatogr. B: Biomed. Sci. Appl., 2001, 761, 247-254.

4 H. Kanazawa, K. Yamamoto, Y. Matsushima, N. Takai, A. Kikuchi, Y. Sakurai and T. Okano, Anal. Chem., 1996, 68, 100-105.

5 C. de Las Heras Alarcon, S. Pennadam and C. Alexander, Chem. Soc. Rev., 2005, 34, 276-285.

6 S. Nayak, H. Lee, J. Chmielewski and L. A. Lyon, J. Am. Chem. Soc., 2004, 126, 10258-10259.

7 R. Hoogenboom, Angew. Chem., Int. Ed., 2009, 48, 7978-7994. 8 M. Sahn, T. Yildirim, M. Dirauf, C. Weber, P. Sungur, S. Hoeppener and U. S. Schubert, Macromolecules, 2016, 49, 7257-7267.

9 D. Schmaljohann, Adv. Drug Delivery Rev., 2006, 58, 16551670.

10 F. D. Jochum and P. Theato, Chem. Soc. Rev., 2013, 42, 7468-7483.

11 D. Kurzbach, M. J. Junk and D. Hinderberger, Macromol. Rapid Commun., 2013, 34, 119-134.

12 J. Herzberger, D. Kurzbach, M. Werre, K. Fischer, D. Hinderberger and H. Frey, Macromolecules, 2014, 47, 7679-7690.

13 T. Steinbach and F. R. Wurm, Biomacromolecules, 2016, 17, 3338-3346.

14 F. Zhang, S. Zhang, S. F. Pollack, R. Li, A. M. Gonzalez, J. Fan, J. Zou, S. E. Leininger, A. Pavia-Sanders, R. Johnson, L. D. Nelson, J. E. Raymond, M. Elsabahy, D. M. Hughes, M. W. Lenox, T. P. Gustafson and K. L. Wooley, J. Am. Chem. Soc., 2015, 137, 2056-2066.
15 T. Steinbach and F. R. Wurm, Angew. Chem., Int. Ed., 2015, 54, 6098-6108.

16 G. Becker, T. A. Marquetant, M. Wagner and F. R. Wurm, Macromolecules, 2017, 50(20), 7852-7862.

17 K. N. Bauer, H. T. Tee, M. M. Velencoso and F. R. Wurm, Prog. Polym. Sci., 2017, 73, 122-161.

18 K. Knop, R. Hoogenboom, D. Fischer and U. S. Schubert, Angew. Chem., Int. Ed., 2010, 49, 6288-6308.

19 H. Lin, T. Wolf, F. R. Wurm and M. A. Kelland, Energy Fuels, 2017, 31, 3843-3848.

20 T. Wolf, T. Steinbach and F. R. Wurm, Macromolecules, 2015, 48, 3853-3863.

21 T. Steinbach, S. Ritz and F. R. Wurm, ACS Macro Lett., 2014, 3, 244-248.

22 H. Wang, L. Su, R. Li, S. Zhang, J. Fan, F. Zhang, T. P. Nguyen and K. L. Wooley, ACS Macro Lett., 2017, 6, 219-223.

23 G. Becker, L. M. Ackermann, E. Schechtel, M. Klapper, W. Tremel and F. R. Wurm, Biomacromolecules, 2017, 18(3), 767-777.

24 A. P. Dove, ACS Macro Lett., 2012, 1, 1409-1412.

25 O. Nuyken and S. D. Pask, Polymers, 2013, 5, 361403.

26 T. Steinbach, R. Schroder, S. Ritz and F. R. Wurm, Polym. Chem., 2013, 4, 4469-4479.

27 Y.-C. Wang, Y. Li, X.-Z. Yang, Y.-Y. Yuan, L.-F. Yan and J. Wang, Tunable Thermosensitivity of Biodegradable Polymer Micelles of Poly( $\varepsilon$-caprolactone) and Polyphosphoester Block Copolymers, Macromolecules, 2009, 42(8), 3026-3032.

28 Y. Iwasaki, T. Kawakita and S.-i. Yusa, Chem. Lett., 2009, 38, 1054-1055.

29 T. Wolf, J. Nass and F. R. Wurm, Polym. Chem., 2016, 7, 2934-2937.

30 T. Wolf, T. Rheinberger and F. R. Wurm, Eur. Polym. J., 2017, 95, 756-765.

31 F. Hofmeister, Arch. Exp. Pathol. Pharmakol., 1888, 24, 247260.

32 M. J. Junk, W. Li, A. D. Schluter, G. Wegner, H. W. Spiess, A. Zhang and D. Hinderberger, Angew. Chem., Int. Ed., 2010, 49, 5683-5687.

33 D. Kurzbach, M. N. Reh and D. Hinderberger, ChemPhysChem, 2011, 12, 3566-3572.

34 M. J. N. Junk, W. Li, A. D. Schlüter, G. Wegner, H. W. Spiess, A. Zhang and D. Hinderberger, Macromol. Chem. Phys., 2011, 212, 1229-1235.

35 V. Bercu, M. Martinelli, C. A. Massa, L. A. Pardi and D. Leporini, J. Chem. Phys., 2005, 123, 174906. 